\title{
3 Technik, bis der Arzt kommt? Was Smart-Health-Lösungen im Gesundheitsbereich leisten können - Und was nicht
}

\author{
Tim Lange
}

Digitale Technik kann immer mehr und ist immer selbstverständlicher Bestandteil unseres Lebens: Mit Fitness-Trackern motivieren wir uns zu mehr Bewegung; sind unsere Vitalwerte schlecht, ziehen wir einen Arztbesuch in Erwägung. Die Chancen der Digitalisierung für die Medizin sind damit aber noch lange nicht ausgeschöpft. Die Möglichkeiten, die Smart Health bietet und vor allem künftig noch bieten wird, werden zu tiefgreifenden gesundheitswirtschaftlichen und gesellschaftlichen Veränderungen führen.

Auf die wichtigsten fünf Entwicklungen, die wir im Bereich Smart Health meiner Meinung nach zu erwarten haben, möchte ich in diesem Beitrag eingehen.

\section{Die fünf zentralen Entwicklungen im Bereich Smart Health}

\section{Quantify yourself - Verbesserung des Gesundheitszustandes über regelmäßige Selbstauswertung}

Schon seit geraumer Zeit existiert eine Vielzahl technischer Geräte am Markt, die im Alltag oder beim Sport Bewegungs- und Gesundheitsdaten aufzeichnen: Sogenannte Wearables, tragbare Minicomputer beispielsweise am Handgelenk, oder SmartphoneApps zeichnen im Alltag oder beim Sport Bewegungs- und Cesundheitsdaten auf, von Schritten über verbrauchte Kalorien bis zu Kreislaufdaten.

Immer öfter richten sich solche Angebote auch an chronisch Kranke wie Diabetiker oder Herz- und Rheumapatienten, und immer mehr Menschen nutzen solche tech- 


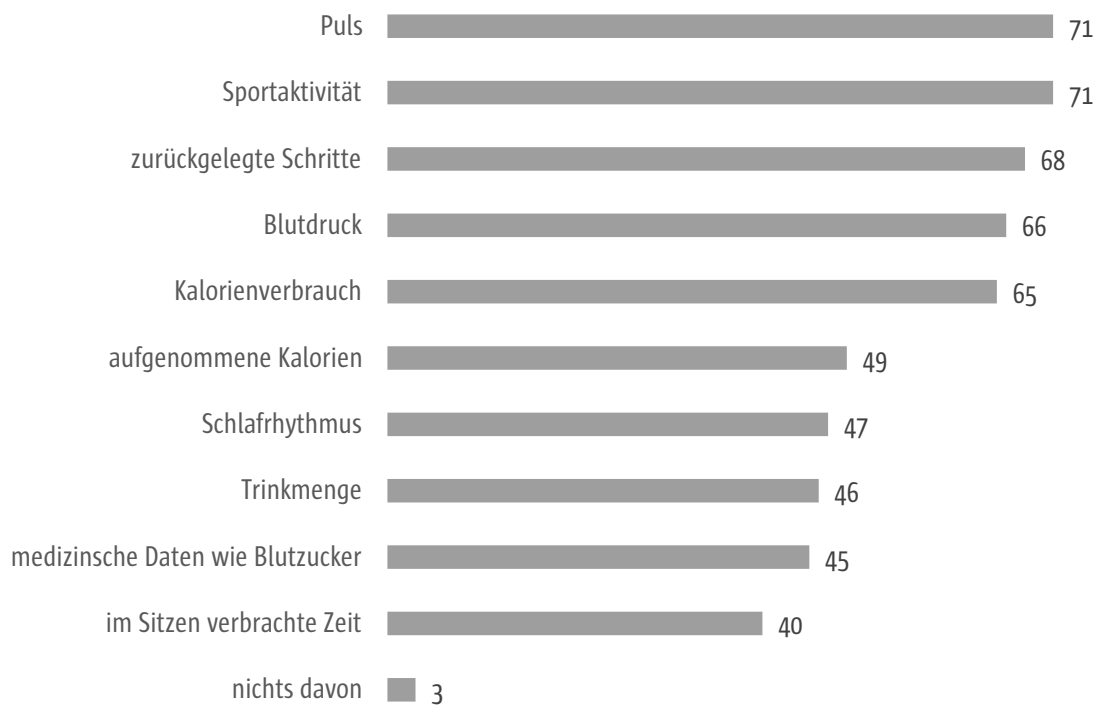

Abb. 1 Vitaldaten sind im Kommen. Welche Daten würden Sie aufzeichnen? (Angaben in Prozent) (\#SmartHealth - Wie smart ist Deutschland?, Techniker Krankenkasse 2016)

nischen Angebote, um ihren Gesundheitsstatus zu verbessern. Die ermittelten Werte geben Orientierung und helfen so zum Beispiel dabei, sich gesund zu ernähren oder abzunehmen. Und sie können helfen, sich auf einen Arztbesuch gut vorzubereiten und „mitreden“ zu können.

Studien - wie beispielsweise die „Smart Health“-Studie der Krankenkasse TK (s. Abb. 1) - zeigen, dass die Bereitschaft, das eigene Gesundheitsverhalten zu dokumentieren, bei Teilen der Bevölkerung vorhanden ist.

Zukunftsvision: eine Bevölkerung, die im Alltag ganz selbstverständlich Gesundheitsdaten über sich selbst sammelt. In zehn Jahren werde es im Alltag üblich sein, einen Fitnesstracker bei sich zu haben, davon ist die große Mehrheit der Befragten in der TK-Studie überzeugt. Dabei werden die Daten, die die Menschen über ihr Verhalten sammeln, immer detaillierter.

Ich erwarte, dass sowohl die Menge und Art der ermittelten Daten steigen wird als auch die generelle Akzeptanz innerhalb der Bevölkerung.

\section{Frühzeitig intervenieren - Gesundheitsstatus aus Metadaten ermitteln}

Neben den „direkt“ gemessenen Werten wie zum Beispiel der Schrittzahl oder dem Blutdruck werden bei der Nutzung von Telekommunikationsmedien jede Menge Metadaten gesammelt - Informationen, die über die eigentlichen Kommunikationsinhalte hinaus anfallen. Da immer mehr Bereiche unseres Lebens digitalisiert werden, fallen auch immer mehr Daten an, aus denen sich - wenn man sie kombiniert zunehmend detaillierte Rückschlüsse auf das Verhalten eines Menschen und damit auch auf dessen Gesundheit schließen lassen. 
Positiv nutzen lassen wird sie sich zum Beispiel im Bereich der Prävention - die Kombination von Bewegungsprofilen, Aktivitätsmessung und Konsumdaten wird dabei helfen, veränderte Gesundheitszustände frühzeitig zu erkennen, sodass wenn nötig ein Arztbesuch erfolgen kann. So kann zum Beispiel eingeschränkte Bewegungsaktivität gepaart mit reduzierter Flüssigkeitsaufnahme und modifiziertem Schlafrhythmus ein möglicher Indikator einer beginnenden Erkrankung sein.

\section{Das Smartphone als Diagnosegerät}

„Husten Sie mal!“ - Eine Standardaufforderung des Arztes, der daraufhin attestiert, ob der Husten fest sitzt, ein trockener oder eher feuchter Husten ist, auf einer Erkältung oder zu viel Zigarettenkonsum beruht. Bestimmte Standard-Diagnosen wie diese dürften künftig zunehmend vom Smartphone gelöst werden. Technisch ist hier bereits viel möglich: So gibt es bereits eine App, die Atemwegserkrankungen elektronisch erkennt - Asthma, Pseudokrupp, Bronchitis oder Lungenentzündung. Kurz ins Mikrophon des Smartphones husten und der Algorithmus der App sagt, woran man wahrscheinlich leidet. Sie wird allerdings derzeit noch getestet, erste Ergebnisse sind aber vielversprechend: Demnach erkennt die App am Klangbild des Hustens die Erkrankung mit Genauigkeiten von mehr als go Prozent.

Mit der Messung von weiteren Parametern werden künftig weitere und umfassende Diagnosen möglich sein, vor allem für bestimmte Standard-Diagnosen. Über einen Anruf beim Hausarzt lässt sich dann entscheiden, wie weiter vorgegangen werden soll - soll der Patient vorstellig werden, zu einem Facharzt gehen oder sich einfach erst einmal ins Bett begeben?

Neben diesem „akustischen“ Beispiel sind bereits heute Anwendungen im Test, die mithilfe der leistungsfähigen Kameras in Smartphones Hauterkrankungen analysieren und erste Hinweise auf die weitere empfohlene Vorgehensweise geben. Darüber hinaus können Krankheits- und Therapieverläufe gezielt zum Beispiel über personalisierte Fragebögen und Gamifizierung verfolgt werden (s. dazu auch Therapieüberwachung).

\section{Telemedizin - Wenn der Arzt virtuell zum Patienten kommt}

Schon heute leiden viele ländliche Gebiete unter einer medizinischen Unterversorgung. Durch den demografischen Wandel wird sich der Mangel an Fachärzten weiter verschärfen. Viele Patienten nehmen lange Wege und Wartezeiten auf sich, um ihre Ärzte zu besuchen. Oft wäre es einfacher, sie könnten ihre Werte selbst messen und online besprechen. Das wäre manchmal auch für den Patienten durchaus wünschenswert: Er müsste nicht mehr für jede kurze Routineauskunft in die Praxis und sich dort gegebenenfalls im überfüllten Wartezimmer hoher Infektionsgefahr aussetzen.

Mit Telemedizin kann eine qualitativ hochwertige Versorgung unabhängig vom Aufenthaltsort der Patienten angeboten werden. Experten sehen dies nicht zuletzt als Beitrag zur Versorgungsgerechtigkeit in unterversorgten Gebieten, vor allem auf dem Land. Bereits heute gibt es zahlreiche Telemedizin-Projekte und viele niedergelassene Ärzte bieten bereits eine Online-Sprechstunde an. Ab Juli 2017 wird diese Leistung auch in die vertragsärztliche Versorgung aufgenommen. Alles, was Arzt und Patient brauchen, sind Computer und Internet und eine gesicherte Datenverbindung, 
Herz- oder Diabetespatienten können so von Zuhause aus Werte wie EKG, Blutdruck, Gewicht oder Blutzucker elektronisch an einen Arzt geben. Der behandelnde Arzt kann die Werte auch ohne ständige Praxisbesuche oder Krankenhausaufenthalt seiner Patienten lückenlos überprüfen. Dazu kommt: Werden Patientendaten in einer Cloud gesammelt, bedeutet dies auch einen extremen Wissenszuwachs: Informationen über einen Patienten gehen nicht mehr verloren, die Zielgenauigkeit von Behandlungen steigt.

Der Telemedizin wird eine enorm steigende Bedeutung zukommen und kann eine Entlastung für Ärzte wie Patienten bedeuten. Können einfache Fragen bereits in einer Videokonferenz beantwortet werden, gibt es schneller freie Termine. Überfüllte Wartezimmer und Notaufnahmen leeren sich. Die Patienten bekommen die Auskunft, die sie brauchen, bequem von zu Hause aus - unabhängig davon, wie weit entfernt sie wohnen. Und die Ärzte können sich in ihrer Praxis auf diejenigen konzentrieren, die persönliche ärztliche Hilfe benötigen.

\section{Therapieüberwachung - Zwei Augen sind nicht mehr genug}

Last but not least wird die Technik die Therapieüberwachung revolutionieren: Je genauer zum Beispiel Bewegungen auch von technischen Geräten überprüft werden können, umso näher rückt die Möglichkeit, dass zum Beispiel die korrekte Ausführung physiotherapeutischer Übungen jederzeit ambulant und ohne Fachpersonal überwacht werden kann.

Auch bei der Therapie von psychischen Problemen soll die Technik zunehmend helfen - von Alltagsstress bis Depressionen, von Prävention bis Therapieersatz. Vorteil: Der Gang zum Psychotherapeuten beispielsweise ist für viele eine Hürde. Apps können hier eine Brücke bauen. Und Patienten mit Ess-Störungen können unter anderem Tagebuch führen, um nicht wieder in ein gestörtes Essverhalten zurückzufallen.

Ob als vorbeugende Maßnahme, Therapiebegleitung oder gar Therapieersatz: Apps sind vor allem eins: kostengünstig. Zudem sind sie ständig verfügbar: So ließe sich mehr Menschen helfen, als es bisher durch traditionelle Therapieangebote möglich ist.

\section{Fazit}

Bereits heute sind E-Health-Angebote eine gute Möglichkeit, die Versorgungsqualität älterer oder chronisch kranker Menschen zu verbessern bzw. Gesunde bei der Vorbeugung von Krankheiten zu unterstützen. Die Entwicklung wird in diesem Bereich in den kommenden Jahren rasant und unaufhaltsam voranschreiten. Die Potenziale von Big-Data-Anwendungen liegen vor allem in der Forschung und Entwicklung sowie in der Effizienzsteigerung bei der Patientenversorgung.

Trotzdem wird auch in Zukunft - zum Glück! - die Digitalisierung den Faktor Mensch im Gesundheitswesen nicht ersetzen. Sie wird vielmehr dabei helfen, mit den Folgen des demografischen Wandels umzugehen, die Gesundheits-Prävention aktiv zu unterstützen und die Versorgung von Patienten zu verbessern. 
3 Technik, bis der Arzt kommt? Was Smart-Health-Lösungen im Gesundheitsbereich leisten können - Und was nicht

Dabei ist für die gesamte Thematik Vertrauen ein zentraler Schlüsselbegriff. Studien wie die TK-Studie Smart Health haben gezeigt, dass zwar kaum Zweifel daran bestehen, dass die Zukunft im Gesundheitswesen auch digital ist. Gleichzeitig bestehen aber auch Vorbehalte gegenüber den Neuerungen, die Smart Health mit sich bringen wird. Begründet sind diese u.a. mit der Sorge um einen nicht ausreichenden Datenschutz.

Hier sind die Akteure des Gesundheitssystems gefordert: Sie müssen den Datenschutz weiterhin sehr ernst nehmen. Es muss noch deutlicher als bisher kommuniziert werden, was getan wird, um Datenschutz zu gewährleisten. Dazu zählt es auch, zu akzeptieren, dass die Menschen selbst bestimmen wollen, welche Daten erhoben werden, wer diese einsehen darf und zu welchem Zweck sie ausgewertet werden und dies dann auch sicherzustellen. 